\title{
Research on the monitoring and adjusting system of power line towers
}

\author{
FuXiang Zhao, a, YiYong Yao ${ }^{2, b}$, AuJun Guo, , JianXun Ma ${ }^{2, b}$,XiangLi Liu, ${ }^{1, b}$, \\ Dong Luo ${ }^{2, b^{*}}$, ,Xing Liu ${ }^{1, b}$, Peng $\mathrm{Li}^{2, \mathrm{~b}}$, Xu Dong Wang ${ }^{1, \mathrm{~b}}$, Peng Dong Wang ${ }^{2, \mathrm{~b}}$ \\ and W W Zhen',b \\ ${ }^{1}$ Shenhua Shendong coal Refco Group Ltd,China \\ 2 Department of civil engineering, Xi'an Jiaotong University, Xi'an 710054, China \\ luodong@xjtu.edu.cn
}

Keywords: Power line towers, testing equipment, risk level, grade evaluation, adjustment device. Abstract. This paper design a set of remote monitoring and testing equipment aimed at the inclination of the transmission tower, and proposed a methodology of evaluating risk degree to evaluate the risk level of the current state of the tower according to the test data. This paper gives a set of adjustment equipment and adjustment method to adjust the transmission tower that have danger of collapse. Finally, this paper gives a method of adjusting transmission tower by PLC software.

\section{Introduction}

With the rapid growth of the economy, the transmission line coverage of the electric power industry have been expanded[1-2]. At the same time, natural disasters such as lightning, storm, and snow coupled with other causes such as the coal mining subsidence area[3-5], poor construction engineering, causing that the transmission line tower become tilted, thus have a negative effect on the national economy, agricultural production and people's life[6-7].

\section{The overall scheme of the system}

The whole system consists of two subsystems: detection system and leveling system. Detection system is divided into two parts: the scene monitoring part and the remote monitoring center. The field monitoring part includes power supply, data acquisition equipment and signal transmission equipment, in which power supply for detection equipment and signal transmission equipment, data acquisition equipment used to detect the tilt of pole tower, signal transmission equipment is used to transmit data to monitoring center for staff to carry out remote analysis. The monitoring center can display the monitoring data on the site through the wireless network on the server of the remote monitoring center and calculate the height of the top of the tower's feet.

The detection system is composed of solar panels, batteries, sensors and signal transmitters. The solar cell is used to charge the battery in the field. It is the most primitive energy for the whole system. Here, the HYXMS4518 of Shenzhen Hongyi Energy Technology Co., Ltd. has the maximum power of 45W, the working current is $2.5 \mathrm{~A}$, and the working voltage is $18 \mathrm{~V}$. The battery stores electrical energy for the sensor and signal transmitter. The battery needs to match the voltage and current of the solar panel and provide enough current and voltage to the sensor and signal transmitter, so that the battery is working properly. Here is the LC-T12105 of the Panasonic. The voltage is $12 \mathrm{~V}$, the rated capacity is $10.5 \mathrm{AH}$. The sensor uses the HXA-SD-V010 dual axis sensor of Tianjin Huaxing Electronic Technology Co., Ltd., its precision is up to 0.01 degrees, and the voltage output mode of 0-10V is used. In order to improve the precision of the detection, the tilt sensor is placed in the higher position of the tower, and the top of the tower is easy to be considered. Wind and other factors affect bending, so it is recommended to be placed at the height of $2 / 3$ of the tower. The signal transmitter sends the signal of the collected sensor to the server by wireless transmission, for the inspector to analyze and monitor the use, select the H7710 CDMA DTU model of the macro electric power, it can receive the signal of the sensor through the RS485 communication and support the two data transmission mode of GPRS/CDMA. 


\section{Establishment and classification of tower collapse risk model}

\section{Establishment of a model of the risk degree of tower collapse}

The main reason for the tower collapse is caused by the torque of the pole and tower, and the force of the tower is mainly the binding force of the wire and its own gravity.

The following example is given to illustrate the process of finding the moment of the tower, which is discussed separately because the process of finding the moment of the tilted tower and the tilted tower is slightly different.

(1) the process of obtaining the resultant moment of the tower without inclination.

As shown in the following figure, it is a three-dimensional force diagram of a tower that has not yet been tilted. With the centre "O" Of the bottom of the tower as the original point, the coordinate system, as shown in the diagram, is subjected to four forces of $F_{1}, F_{2}, F_{3}$, and $F_{4}$. The direction of $F_{1}$ and $F_{3}$ is in the negative direction and the direction of the $X$ axis respectively, the $F_{2}$ and $X$ axis, the $Y$ axis, and the $\mathrm{Z}$ axis are alpha, respectively, respectively. The angles of $\gamma_{1}, \mathrm{~F}_{4}$ and $\mathrm{X}$ axis, $\mathrm{Y}$ axis and $\mathrm{Z}$ axis are $\alpha_{1}$, $\beta_{2}$ and $\gamma_{2}$, respectively.

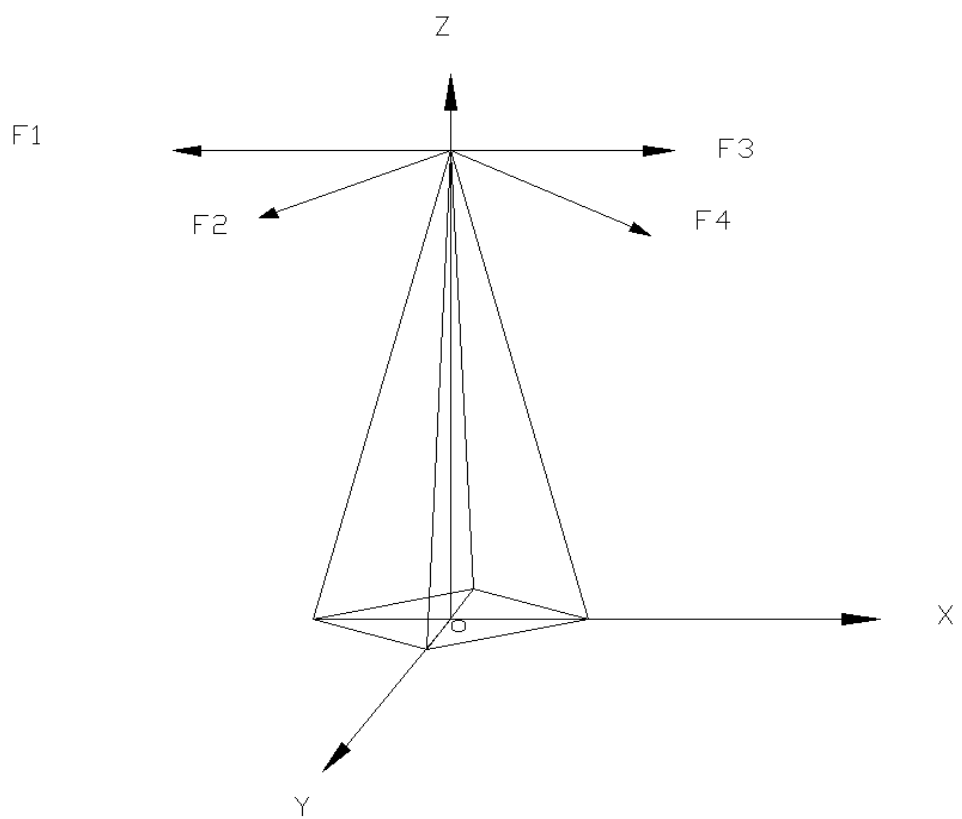

Figure 1. Three-dimensional stress of a tower without a tilt.

a. Each pole of a tower is decomposed into a force vector along three axes:

$$
\begin{aligned}
& F_{2 x}=F_{2} * \cos \alpha_{1} \\
& F_{2 y}=F_{2} * \cos \beta_{1} \\
& F_{2 z}=F_{2} * \cos \gamma_{1} \\
& F_{4 x}=F_{4} * \cos \alpha_{2} \\
& F_{4 y}=F_{4} * \cos \beta_{2} \\
& F_{4 z}=F_{4} * \cos \gamma_{2}
\end{aligned}
$$

b. The binding force of all wires obtained in the previous step is summed up, and the binding force of all wires to the tower is obtained in three axes:

$$
\begin{gathered}
F_{x}=F_{3}+F_{4} * \cos \alpha_{2}-F_{2} * \cos \alpha_{1}-F_{1} \\
F_{y}=F_{2}=\cos \beta_{1}+F_{4} * \cos \beta_{2} \\
F_{z}=F_{2} * \cos \gamma_{1}+F_{4} * \cos \gamma_{2}
\end{gathered}
$$

c. The resultant moments in three directions are obtained based on the moment formula $\mathrm{M}=\mathrm{F}^{*} \mathrm{~L}$ :

$$
M_{x}=F_{x} * h=\left(F_{3}+F_{4} * \cos \alpha_{2}-F_{2} * \cos \alpha_{1}-F_{1}\right) * h
$$




$$
M_{y}=F_{y} * h=\left(F_{2} * \cos \beta_{1}+F_{4} * \cos \beta_{2}\right) * h
$$

$\mathrm{M}_{\mathrm{z}}=0$ (because the tower is not tilted, the gravity force arm in the $\mathrm{Z}$ direction is 0 , that is, the torque is constant 0 ).

d. Vector sum is made for the resultant moments in three directions, and the total moment of the tower is obtained.

$$
\begin{aligned}
& M_{\mathrm{A}}=\sqrt{M_{x}^{2}+M_{y}^{2}+M_{x}^{2}} \\
& =\sqrt{\left(\left(F_{3}+F_{4} * \cos \alpha_{2}-F_{2} * \cos \alpha_{1}-F_{1}\right) * h\right)^{2}+\left(\left(F_{2} * \cos \beta_{1}+F_{4} \cdot \cos \beta_{2}\right) * h\right)^{2}}
\end{aligned}
$$

(2) the process of obtaining the resultant moment of an inclined tower.

When the tilt angle of the tower is $\delta$ and the distance between the centre of the pole tower and the bottom of the tower is $h$, the force of the $F_{1}, F_{2}, F_{3}$ and $F_{4}$ is constant on the three axis, only the force of the force changes, so the resultant moment of the three directions is the moment of force.

$$
\begin{gathered}
M_{x}=F_{x} * h=\left(F_{3}+F_{4} * \cos \alpha_{2}-F_{2} * \cos \alpha_{1}-F_{1}\right) * h * \sin \delta \\
M_{y}=F_{y} * h=\left(F_{2} * \cos \beta_{1}+F_{4} * \cos \beta_{2}\right) * h * \sin \delta \\
M_{x}=F_{x} * h * \cos \delta+\mathrm{G} * h_{z} * \cos \delta
\end{gathered}
$$

The resultant force moment of the tower can be obtained from the above results

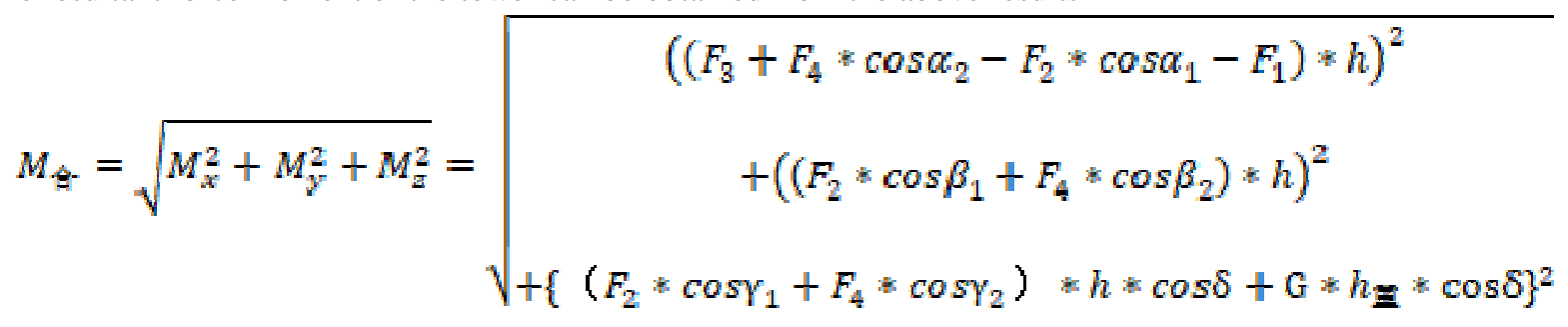

The tower is safe in a certain range of resultant force moments. As the tower is increased by the moment of joint force, the risk of the tower collapse will have a transitional stage. After the transition stage, the possibility of the tower collapse is greatly improved. Therefore, the safety zone passes through the transition zone to the dangerous area. Finally, when the tower is tilted to a certain level, the tower collapses. The curve should be conformed to a tangent curve, assume that the maximum moment that the tower can withstand is $\mathrm{M}_{\max }$. When the tower is subjected to a torque at 0 , the risk is 0 . The risk degree of the tower is $\tan \frac{\pi}{2}$ when the torque reaches $\mathbf{M}_{\max }$ (that is, the tower will collapse), the function image of the risk degree $\mathrm{f}(\mathrm{x})=\tan \left(\frac{M_{4}}{M_{\max }} * \frac{\pi}{2}\right)$.

\section{Classification of risk degree of tower collapse}

The danger degree of tower collapse is divided into 4 stalls, from the diagram of danger degree of tower collapse (as shown).It can be seen that the slope of the first tower is relatively slow before $\frac{M_{\max }}{2}$, that is, the danger degree increases slowly, so the danger degree of the tower is divided into safety zone, and the risk degree of pole tower is moderate in $\frac{M_{\mathrm{max}}}{2}$ to $\frac{3 M_{\mathrm{max}}}{4}$, and the risk of pole tower increases rapidly after $\frac{3 M_{\max }}{4}$. The transition is obvious, so $\frac{M_{\max }}{2}$ to $\frac{3 M_{\max }}{4}$ is divided into transition zone, and $\frac{2 M_{\max }}{4}$ and $M_{\max }$ is divided into safety zone, and the tower will collapse when it exceeds $M_{\max }$, so it is divided into collapsed area. 


\section{Leveling system}

The leveling system is the hydraulic drive mode. It is mainly composed of energy equipment, executive parts and control system. The following is a detailed introduction to each part.

\section{Energy equipment}

Because the system is working outdoors, and the leveling system itself has a large power, the use of batteries is obviously unable to meet the demand for electricity and power, so the generator is used here. Considering that the gasoline generator has the advantages of small size, light weight and good starting property, it is suitable for outdoor carrying and will not be difficult to start in winter, so gasoline generator is used to supply power supply for leveling device. Here is YAMAHA's QF5.0-A (ZSG6000), its working voltage is $380 \mathrm{~V}$, the frequency is $50 / 60 \mathrm{~Hz}$, the rated output is $5.0 \mathrm{KVA}$.

\section{Execution unit}

The executive part is driven by hydraulic cylinder, and the following is its hydraulic schematic diagram. When the motor works, the hydraulic pump will pump out the hydraulic oil through the filter and drive each jack to work. The hydraulic oil goes back to the hydraulic cylinder through the way back to the oil, and when the pressure is too high, the hydraulic oil is back to the hydraulic cylinder through the safety valve.

\section{Control system}

PLC has user oriented instructions such as timing, counting and arithmetic operations. And control various types of mechanical or production processes through digital or analog input / output, and it can operate reliably and without fault for a long time in a bad environment. And easy to connect, easy to maintain, good isolation, strong corrosion resistance, can adapt to a wider range of temperature change, the average time interval of no fault (MTBF) more than 15 years. Therefore, it can not only meet the control functions of the system, but also adapt to the harsh requirements of outdoor equipment. Here is a selection of Taiwan Yonghong FBS-40MAT2-AC, because of its insufficient I/O port, two FBS-6AD modules are added to expand the I/O port.

In addition, in order to display the driving height of the driving part in real-time display, and more humanized operation, the HMI operation panel is added to the system to input the drive part height and the displacement of the real time display driver. Here is the selection of the Kunlun state of TPC1262HI, it is a set of advanced embedded integrated touch screen based on advanced Cortex-A8 CPU (main frequency $600 \mathrm{MHz}$ ). The product is designed to use a 12.1 inch high brightness TFT LCD (resolution $800 \times$ 600), a four wire resistance touch screen (resolution $4096 \times$ 4096), and a good electromagnetic shielding, beautiful and sturdy aluminum alloy structure. At the same time, MCGS Embedded Configuration Software (run version) is pre-installed, which has powerful function of image display and data processing.

\section{Software design}

The software design of the system is divided into two parts: the interface design and the control flow design. The system software design interface.

In the software interface, the user can see the current pressure and height value of the system, which can also set the height of the system to top up, a few steps to achieve the required lift height and the time interval between each step. In addition, considering the safety problem, overload indication is added in the design. When the pressure of the system is greater than the safe range, the overload indicating lamp will give a hint.

The flow chart of stepwise equal proportional jack up. At the beginning, the leveling control system is opened, and setting height, step number and waiting time of each jack are input in the software interface of the touch screen. Then start the pump station and click the up (or down) button at the software interface. When the pressure is not overloaded, the Jack begins to move. During the procession of the jack, the pressure value of the appropriate detection system and the displacement 
value of the sensor are been measured. If overload occurs, alarm immediately and end the process. If there is no alarm state, it is known that the displacement of the jack reaches the set value before it ends.

\section{Acknowledgements}

This work was supported by Research on automatic leveling system of power tower poles and legs in goaf of Shendong Coal Group by China Shenhua Group Corporation(20160114), National Natural Science Foundation of China (Nos. 51508460), Central University's special research funding special fund cross disciplinary project (xjj2017175), Natural Science Foundation Research Program of Shaanxi Province-Youth Talents Project (2016JQ5017), National Post-Doctoral Science Foundation (No. 2015M570838), Shaanxi Province Post-Doctoral Research Fund (2016BSHB083),Shaanxi Province Post-Doctoral supporting research project(2015SXBHPT112), Research Fund Project of Xi'an Jiaotong University.

\section{References}

[1] Dj.M. Maric, P.F. Meier and S.K. Estreicher: Mater. Sci. Forum Vol. 83-87 (1992), p. 119

[2] M.A. Green: High Efficiency Silicon Solar Cells (Trans Tech Publications, Switzerland 1987).

[3] Y. Mishing, in: Diffusion Processes in Advanced Technological Materials, edtied by D. Gupta Noyes Publications/William Andrew Publising, Norwich, NY (2004), in press.

[4] G. Henkelman, G.Johannesson and H. Jónsson, in: Theoretical Methods in Condencsed Phase Chemistry, edited by S.D. Schwartz, volume 5 of Progress in Theoretical Chemistry and Physics, chapter, 10, Kluwer Academic Publishers (2000).

[5] R.J. Ong, J.T. Dawley and P.G. Clem: submitted to Journal of Materials Research (2003)

[6] P.G. Clem, M. Rodriguez, J.A. Voigt and C.S. Ashley, U.S. Patent 6,231,666. (2001)

[7] Information on http://www.weld.labs.gov.cn 